\title{
The functional state of physically gifted preschoolers with high aerobic capacity
}

\author{
Igor A. Krivolapchuk ${ }^{1 *}$, Maria B. Chernova ${ }^{1}$, Liudmila I. Larionova ${ }^{2}$, Vladimir M. \\ Postavnev $^{2}$, and Sergey $A$. Kesel ${ }^{3}$ \\ ${ }^{1}$ Institute of Developmental Physiology, Russian Academy of Education, Laboratory of Physiology of \\ Muscular Activity and Physical Education, Moscow, Russia \\ ${ }^{2}$ Moscow City University, Institute of Pedagogics and Psychology of Education, Department of \\ Psychology, Moscow, Russia \\ ${ }^{3}$ Yanka Kupala State University of Grodno, Department of Sports Disciplines, Grodno, Belarus
}

\begin{abstract}
The study aims to identify the features of the functional state of physically gifted children 5-6 years old with a high level of development of aerobic capacity under conditions of cognitive loads performed at different speeds. The authors used tests based on Anfimov's letter tables as models of intense cognitive load. The examination was carried out at rest and while working at a comfortable speed and the maximal possible speed. Based on the results of the tasks, the speed and productivity of work, indicators of the psychophysiological cost of activity were calculated. Using the Luscher test, the level of situational anxiety was assessed and the coefficient of autonomic tone was determined. To diagnose the emotional state, the Lutoshkin technique was used. The tension of the systems of autonomous and central regulation of physiological functions was measured based on temporal analysis of the heart rhythm. Systolic and diastolic blood pressure, pulse and mean pressure, double product, Kerdo vegetative index, Myznikov index, and functional changes index were also calculated. A complex indicator of the development of aerobic capacity was found, taking into account the power, capacity, and efficiency of an aerobic source of energy supply to muscles. Based on the sigma scale, 7 gradations for assessing the development of aerobic abilities were identified, namely, very low, low, below average, average, above average, high, very high (above $\mathrm{M}+2.0 \sigma$ ). The results obtained show that psychophysiological changes in the functional state during intense cognitive load in children aged 5-6 years largely depend on the development of aerobic capacity. It was found that physically gifted preschoolers with a high level of development of aerobic capacity were distinguished by optimally reduced activity during a calm wakeful state and less pronounced psychophysiological reactivity in conditions of intense cognitive load. They were characterized by increased efficiency of activity and its low psychophysiological cost in combination with increased adaptive potential of the organism.
\end{abstract}

\footnotetext{
*Corresponding author: i.krivolapchuk@mail.ru
} 
Keywords: gifted children, aerobic ability, developmental level, functional state.

\section{Introduction}

There are three main functional abilities of a person that determine their physical performance: alactate anaerobic capacity, glycolytic anaerobic capacity, and aerobic capacity [1]. Aerobic capacity provides the ability to perform physical work by enhancing aerobic processes in tissues with a simultaneous increase in the activity of the oxygen transport system, which delivers oxygen to working muscles [1]. Since aerobic capacity is largely genetically determined [2], it serves as a physiological criterion for assessing a person's predisposition to efficiently performing work associated with the manifestation of general endurance. Along with this, individuals with well-developed aerobic capacity have an increased resistance to psychosocial stress [3-7], high cognitive opportunities, and academic performance [8-11]. They are characterized by increased power and efficiency of functioning of stress-implementing and stress-limiting systems, which is manifested in the optimal functional state (FS) and a reduced biological cost of adaptation to adverse influences [3, 4, 12-14]. At the same time, today one can note a shortage of strictly verified scientific information regarding the influence of the degree of development of aerobic ability on the psychophysiological reactivity of preschool children under intense cognitive loads.

The study aims to reveal the features of FS of physically gifted children at 5-6 years with a high level of development of aerobic capacity with cognitive loads performed at different speeds.

\section{Methods}

The study involved healthy preschoolers aged 5-6 years $(n=209)$.

We used tests based on Anfimov's letter tables as models of intense cognitive load. The examination was carried out at rest and while working at a comfortable speed and the maximal possible speed. Based on the results of the tasks, speed (A) and work productivity (Q) were calculated. Using the Luscher test, the level of situational anxiety (SA) was assessed and the vegetative tone coefficient (VTC) was determined. To diagnose the emotional state (ES), the Lutoshkin technique was used.

To assess the tension of the systems of autonomous and central regulation, time-lapse analysis of the heart rate was used. Peak heart rate (HR), mean duration of R-R interval (RRNN), standard deviation (SDNN), spread of cardio intervals (MxDMn), mode (Mo), the amplitude of mode (AMo), stress index (SI) were determined.

Systolic (SP) and diastolic (DiP) blood pressure was recorded, pulse (PP) and mean pressure (MAP), double product (DP), Kerdo vegetative index (KVI), Myznikov index (MI), and functional changes index $(\mathrm{FCI})$ were calculated. The psychophysiological cost of activity was determined based on calculating the following ratios: $\mathrm{Q} / \Delta \mathrm{HR}, \mathrm{Q} / \Delta \mathrm{SI}, \mathrm{Q} / \Delta \mathrm{DP}, \mathrm{A} / \Delta \mathrm{HR}$, $\mathrm{A} / \Delta \mathrm{SI}, \mathrm{A} / \Delta \mathrm{DP}$.

A complex indicator of the development of aerobic capacity was found, taking into account power (PWC170), capacity (coefficient B of the Muller equation), and efficiency (watt-pulse, WP) of an aerobic source of muscle energy supply. Based on the sigma scale, 7 gradations for assessing the development of aerobic abilities were identified: very low, low, below average, average, above average, high, very high (above $M+2.0 \sigma$ ). 


\section{Results and discussion}

Comparison of FS indices in children aged 5-6 years in a calm wakeful state revealed significant differences $(\mathrm{p}<0.05)$ due to the development of aerobic capacity. Physically gifted preschoolers with a very high level of aerobic performance differed from children with a very low level of aerobic capacity by higher RRNN values and lower values of HR, FCI, VTC, ES. Differences between children with very high and medium levels of aerobic capacity were less pronounced and related to HR and ES. In general, these data indicate an optimally reduced background activity in preschoolers with a well-developed aerobic capacity, combined with a high adaptive potential of the organism.

Working with a cognitive load at a comfortable speed caused statistically significant ( $\mathrm{p}$ $<0.05-0.001$ ) changes in the majority of FS indices in children aged 5-6 years compared with the resting state (Fig. 1). Multidirectional shifts in SP, DiP, MAP, RRNN, Mo, AMo, SDNN, MxDMn, SI, DP, KVI, MI, ST, VTC were observed, indicating a significant increase in the level of nonspecific activation, an increase in the activity of the sympathetic division of the autonomic nervous system (ANS) and inhibition of the activity of the parasympathetic division, an increase in the influence of the mechanisms of central regulation on the HR and hemodynamics.

Working with the maximum speed caused even more significant changes in the studied parameters of the FS ( $\mathrm{p}<0.01-0.001)$ in comparison with a calm wakeful state (see Fig. 1). Only the shifts in the speed indicator (A) during operation at the maximum pace concerning the load in the auto-pace mode were insignificant.

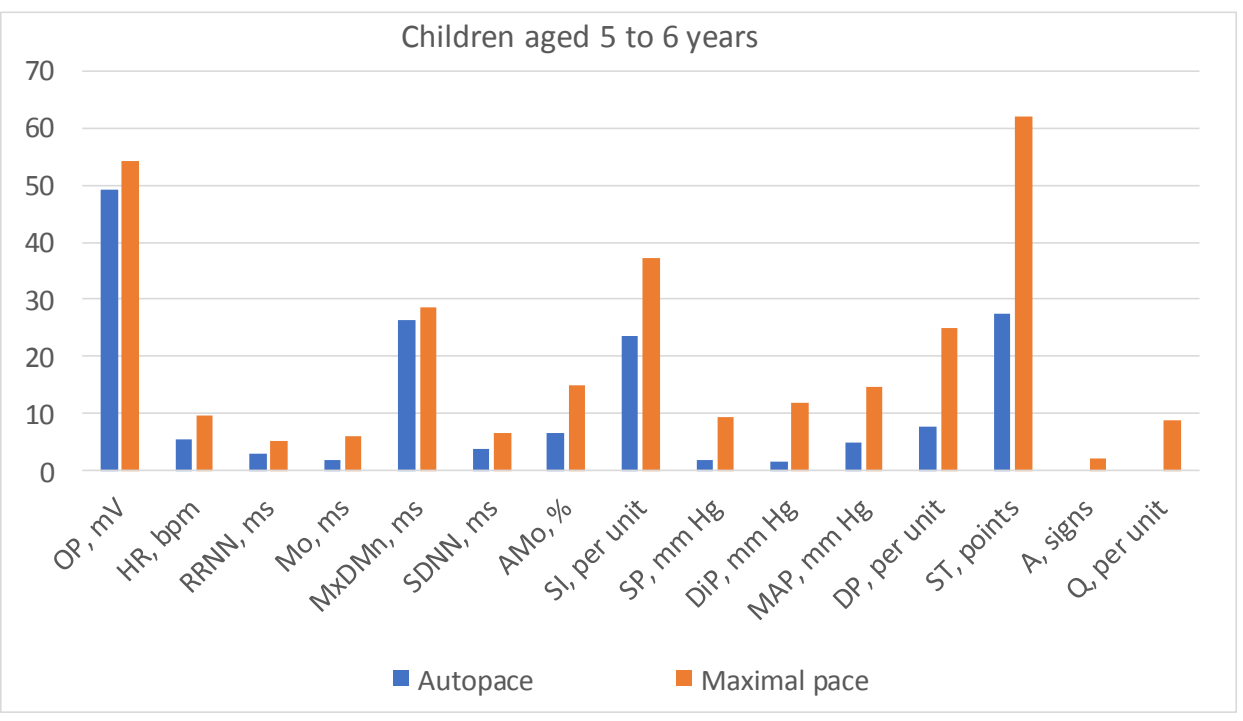

Fig. 1. Shifts (\%) of FS indicators during working with the load in comparison with the calm wakeful state

Comparison of changes in performance under different load modes showed that the number of viewed signs (A) increased when working at maximum pace, and productivity (Q), on the contrary, tended to decrease. The change in the operating mode was accompanied by an increase in the psychophysiological cost of the activity. This is reflected in a decrease in $\mathrm{Q} / \Delta \mathrm{HR}, \mathrm{Q} / \Delta \mathrm{SI}, \mathrm{Q} / \Delta \mathrm{DP}$, as well as, in some cases, $\mathrm{A} / \Delta \mathrm{HR}, \mathrm{A} / \Delta \mathrm{SI}, \mathrm{A} / \Delta \mathrm{DP}$.

The analysis of individual differences showed that during the performance of test loads in children of 5-6 years with a very high level of development of aerobic capacity, less 
pronounced changes in the considered FS indicators were observed in combination with increased productivity of activity compared to preschoolers with "insufficient" and "average" aerobic productivity. Thus, when working in the auto-pace mode, children with high aerobic capacity differed from children with a very low level of aerobic performance in lower values of peak HR, AMo, DiP, DP, MI, FCI, ST, and VTC and higher values in RRNN, Mo, MxDMn, A/ $\Delta \mathrm{HR}, \mathrm{Q}, \mathrm{Q} / \Delta \mathrm{HR}, \mathrm{Q} / \Delta \mathrm{SI}$. Differences were revealed between preschoolers with very high and medium levels of aerobic performance, concerning HR, DP, and higher values of RRNN, A/ $\Delta \mathrm{HR}, \mathrm{Q} / \Delta \mathrm{HR}$.

Some statistically significant intergroup differences were also observed when working at the maximum pace: physically gifted children with high aerobic ability differed from preschoolers with a very low level of development of that ability demonstrating lower values of peak HR, DiP, DP, MI, VTC, and higher values of Mo, MxDMn, A/ $\Delta \mathrm{HR}, \mathrm{Q} / \Delta \mathrm{SI}$. When comparing children with very high and medium levels of aerobic capabilities, less pronounced differences were observed in the ratio of HR, DP, MI, and MxDMn, A/ $\Delta \mathrm{HR}$.

The results obtained show that physically gifted preschoolers of 5-6 years with a high level of development of aerobic ability under conditions of intense cognitive load are characterized by increased efficiency of activity against the background of an optimally reduced level of nonspecific activation and a shift in the autonomic balance towards an increase in the activity of the parasympathetic division of the ANS, which is in good agreement with the results of other works [5; 13]. It is important to note that in individuals with high aerobic capacity, a shift in the autonomic balance towards an increase in the activity of the parasympathetic division of the ANS is usually combined with an increase in stress resistance $[3 ; 5 ; 7 ; 9]$. It should be emphasized that the found differences depend not only on the genotype but are largely due to the influence of aerobic exercises.

Table 1. Differences between children of 5-6 years with different levels of aerobic capacity development.

\begin{tabular}{|c|c|c|c|}
\hline \multirow{3}{*}{ Criterion } & \multicolumn{3}{|c|}{ Indicators } \\
\hline & \multirow{2}{*}{ Background } & \multicolumn{2}{|c|}{ Cognitive load } \\
\hline & & Autopace & Maximal pace \\
\hline \multicolumn{4}{|c|}{ Differences between groups with very high and very low levels of aerobic capacity } \\
\hline $\begin{array}{c}\text { Aerobic } \\
\text { capacity, per } \\
\text { unit }\end{array}$ & $\begin{array}{l}\text { RRNN, HR, FCI, } \\
\text { KW, ES }\end{array}$ & 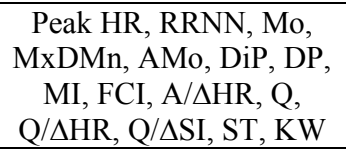 & 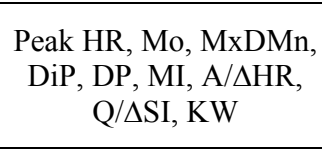 \\
\hline \multicolumn{4}{|c|}{$\begin{array}{c}\text { Differences between groups with very high and medium levels of aerobic } \\
\text { capacity }\end{array}$} \\
\hline $\begin{array}{c}\text { Aerobic } \\
\text { capacity, per } \\
\text { unit }\end{array}$ & HR, ES & $\begin{array}{l}\text { Peak HR, RRNN, DP, } \\
\text { A/ } / \mathrm{HR}, \mathrm{Q} / \Delta \mathrm{HR}\end{array}$ & $\begin{array}{l}\text { Peak HR, DP, MI, } \\
\text { MxDMn, A/AHR }\end{array}$ \\
\hline
\end{tabular}

Note: Here we present statistically significant inter-group differences $(\mathrm{p}<0,05-0,01)$

\section{Conclusion}

The results obtained show that psychophysiological changes in FS during work with an intense cognitive load in children aged 5-6 years largely depend on the development of aerobic capacity.

It was found that physically gifted preschoolers with a high level of development of aerobic capacity were distinguished by optimally reduced activity during a calm wakeful state and less pronounced psychophysiological reactivity in conditions of intense cognitive load. They were characterized by increased efficiency of activity and its low 
psychophysiological cost in combination with increased adaptive potential of the organism. For a more accurate assessment of the influence of aerobic capacity on the FS of children, in conditions of intense cognitive activity, in the future, it is necessary to conduct special longitudinal studies.

\section{Acknowledgments}

This study was supported by the Russian Foundation for Basic Research (RFBR) grant (project No. 19013-00127).

\section{References}

1. N.I. Volkov, V.I. Oleinikov, Bioenergetika sporta [Bioenergetics of sport] (Sovetskii sport, Moscow, 2011). Accessed on: December 20, 2020. [Online]. Available: https://www.elibrary.ru/item.asp?id=21260876

2. L.P. Sergienko, Osnovy sportivnoi genetiki [The basics of sports genetics] (Vishcha shkola, Kyiv, 2004)

3. B.L. Alderman, S.M. Arent, D.M. Landers, T.J. Rogers, Psychophysiology, 44(5), 759-766 (2007). https://doi.org/10.1111/j.1469-8986.2007.00548.x

4. U. Rimmele, Psychoneuroendocrinology, 34, 190-198 (2009). https://doi.org/10.1016/j.psyneuen.2008.08.023

5. G. Everly, Clinical guide to the treatment of the human stress response (SpringerVerlag, NY, 2013). https://doi.org/10.1007/978-1-4614-5538-7

6. S. Klaperski, Psychology of Sport \& Exercise, 14(2), 266-274 (2013). https://doi.org/10.1016/j.psychsport.2012.11.003

7. M.J. Lambiase, Am J Hypertens 26(3), 409-415 (2013). https://doi.org/10.1093/ajh/hps036

8. M.W. Voss, L. Chaddock, J.S. Kim, M. Vanpatter, M.B. Pontifex, L.B. Raine, N.J. Cohen, C.H. Hillman, A.F. Kramer, Neuroscience, 199, 166-176 (2011). https://doi.org/10.3389/fnhum.2014.00584

9. G. Lees, Prev Chronic Dis 10, E174 (2013). https://doi.org/10.5888/pcd10.130010

10. L. Chaddock-Heyman, Dev CognNeurosci 20, 52-58 (2016). https://doi.org/10.1016/j.den.2016.07.001

11. J.E. Donnelly, Med Sci Sports Exerc 48(6), 1223-1224 (2016). https://doi.org/10.1249/MSS.0000000000000901

12. M.S. Sothmann, Psychobiology of physical activity (Human Kinetics Publishers, Champaign, 2006). Accessed on: December 20, 2020. [Online]. Available: https://psycnet.apa.org/record/2006-02237-010

13. R.P. Sloan, P.A. Shapiro, R.E. DeMeersman, E. Bagiella, E.N. Brondolo, P.S. McKinley, I. Slavov, Y. Fang, M.M. Myers, Am J Public Health 99, 921-928 (2009). https://doi.org/10.2105/AJPH.2007.133165

14. C.J. Huang, H.E Webb., K.N. Beasley, D.A. McAlpine, S.E. Tangsilsat, E.O. Acevedo, Appl PhysiolNutrMetab 39(3), 375-380 (2014). https://doi.org/10.1139/apnm-20130297

15. A.K. Moskatova, Fiziologiya Cheloveka, 18, 368-382 (2001). Accessed on: December 20, 2020. [Online]. Available: https://www.elibrary.ru/author_items.asp 
16. N. Armstrong, Res Q Exerc Sport 88(2), 130-148 (2017).

https://doi.org/10.1080/02701367.2017.1303298 